\title{
Genomics in aquaculture to better understand species biology and accelerate genetic progress
}

\author{
José M. Yáñez ${ }^{1,2 *}$, Scott Newman ${ }^{3}$ and Ross D. Houston ${ }^{4}$ \\ ${ }^{1}$ Faculty of Veterinary and Animal Sciences, University of Chile, Santiago, Chile, ${ }^{2}$ Aquainnovo, Puerto Montt, Chile, ${ }^{3}$ Genus \\ plc, Hendersonville, TN, USA, ${ }^{4}$ The Roslin Institute and Royal (Dick) School of Veterinary Studies, University of Edinburgh, \\ Midlothian, UK
}

Keywords: aquaculture, genome, breeding programs, QTL, single nucleotide polymorphisms, next-generation sequencing

The production of fish and shellfish through aquaculture is an increasingly important source of high-quality animal protein, with a worldwide production of 66.6 million tons in 2012 (FAO, 2014). Considering the continuously growing global human population and increasing demand for fish products, improvements in the scale, efficiency, and sustainability of aquaculture are essential. To achieve this, several challenges facing the culture of fish and shellfish species need to be overcome. These relate to the diverse biology of the cultured species and their interaction with environmental factors. Examples include outbreaks of infectious diseases, control of sexual maturation, sustainable feed for carnivorous species, and tolerance of diverse and changing environments. This "Frontiers in Livestock Genomics" Research Topic highlights the opportunities offered by recent developments in the field of genomics, and in particular high-throughput sequencing, to contribute to addressing these challenges, with a focus on selective breeding programmes.

The use of selective breeding as a tool to improve the biological efficiency of production in aquaculture generally lags behind plant and farm animal industries, and less than $10 \%$ of aquaculture production is based on genetically-improved stocks (Gjedrem et al., 2012). Encouragingly, annual genetic gains reported for aquatic species are in general substantially higher than that of terrestrial farm animals (Gjedrem et al., 2012) and there is considerable scope for achieving significant

Edited and reviewed by: Max F. Rothschild,

lowa State university, USA

${ }^{*}$ Correspondence: José M. Yáñez, jmayanez@uchile.cl

Specialty section:

This article was submitted to Livestock Genomics, a section of the journal Frontiers in Genetics

Received: 10 February 2015 Accepted: 17 March 2015 Published: 01 April 2015

Citation:

Yáñez JM, Newman S and Houston $R D$ (2015) Genomics in aquaculture to better understand species biology and accelerate genetic progress.

Front. Genet. 6:128

doi: 10.3389/fgene.2015.00128 positive economic impact via improved breeding schemes. However, the status of breeding programs and the level of technology used for aquatic species production are wide-ranging, from use of wild seed stocks through to family-based selection incorporating genomic tools. Family selection and genomic tools can be applied to improve traits that are expensive or difficult to measure on the selection candidates themselves including disease resistance (Yáñez et al., 2014; Ødegård et al., 2014), flesh color (Colihueque and Araneda, 2014; Ødegård et al., 2014) and other appearance traits such as body shape and skin pigmentation (Colihueque and Araneda, 2014) in finfish species. In contrast, despite the global importance of mollusc species for aquaculture, few selective breeding programmes exist and the state of genomic tools and knowledge for these species is typically lacking (Astorga, 2014).

Genomics resources such as whole genome reference sequences, high-density SNP genotyping arrays and genotyping-by-sequencing are in development for several aquaculture species. Fuller characterisation of these resources is underway and is resulting in improved fundamental knowledge of the genome structure and biology, highlighted in this issue by the analysis of repeat elements in the Asian sea bass genome (Kuznetsova et al., 2014). These resources will provide powerful tools for the research community and will aid in the determination of the genetic factors involved in the regulation of complex traits. For example, high-throughput RNA sequencing can give a holistic view of the host response to infectious diseases, and help identify the important genes and pathways defining genetic resistance, as demonstrated in this issue for 
rainbow trout (Ali et al., 2014; Marancik et al., 2014) and panaeid shrimp (Santos et al., 2014). Sequencing technology has also facilitated the development of abundant genetic markers that have multi-faceted applications for selective breeding of aquatic species, including parentage assignment in mixedfamily environments, providing greater control over family representation and inbreeding (Vandeputte and Haffray, 2014). Medium or high-density SNP arrays can be used to predict genomic breeding values for economically-important traits in well-developed breeding programmes, such as Atlantic salmon (Ødegård et al., 2014). For instance, based on simulations of a Pacific white shrimp breeding program, genetic progress of disease resistance traits is faster with genomic-enabled selection compared to conventional phenotype-based selection due to higher accuracy (Castillo-Juárez et al., 2015). Incorporation of genetic marker information can also be a useful asset to optimize genetic diversity and future genetic gain when establishing base populations for breeding programmes (Fernández et al., 2014). Furthermore, these genomic tools can be applied to investigate putative genomic signatures of selection during the domestication process of farmed fish species, thus potentially identifying genomic regions underlying variation in relevant phenotypes in wild and domestic fish populations (López et al., 2015).

Aquaculture species typically have several common features, for example high fecundity and external fertilization, plus a short evolutionary distance from their wild ancestors. The reproductive features enable flexible mating structures to be used for breeding programmes, and can provide a powerful resource for genetic studies of complex traits, such as disease resistance (Yáñez et al., 2014). However, the diversity between these species is enormous and often necessitates the establishment

\section{References}

Ali, A., Rexroad, C. E., Thorgaard, G. H., Yao, J., and Salem, M. (2014). Characterization of the rainbow trout spleen transcriptome and identification of immune-related genes. Front. Genet. 5:348. doi: 10.3389/fgene.2014.00348

Astorga, M. P. (2014). Genetic considerations for mollusk production in aquaculture: current state of knowledge. Front. Genet. 5:435. doi: 10.3389/fgene.2014.00435

Castillo-Juárez, H., Campos-Montes, G. R., Caballero-Zamora, A., and Montaldo, H. H. (2015). Genetic improvement of Pacific white shrimp (Penaeus (Litopenaeus) vannamei): perspectives for genomic selection. Front. Genet. 6:93. doi: 10.3389/fgene.2015.00093

Colihueque, N., and Araneda, C. (2014). Appearance traits in fish farming: progress from classical genetics to genomics, providing insight into current and potential genetic improvement. Front. Genet. 5:251. doi: $10.3389 /$ fgene. 2014.00251

FAO. (2014). The State of World Fisheries and Aquaculture Opportunities and challenges. Rome: FAO, 243.

Fernández, J., Toro, M. Á., Sonesson, A. K., and Villanueva, B. (2014). Optimizing the creation of base populations for aquaculture breeding programs using phenotypic and genomic data and its consequences on genetic progress. Front. Genet. 5:414. doi: 10.3389/fgene.2014.00414

Gjedrem, T., Robinson, N., and Rye, M. (2012). The importance of selective breeding in aquaculture to meet future demands for animal protein: a review. Aquaculture 350, 117-129. doi: 10.1016/j.aquaculture.2012.04.008

Kuznetsova, I. S., Thevasagayam, N. M., Sridatta, P. S. R., Komissarov, A. S., Saju, J. M., Ngoh, S. Y., et al. (2014). Primary analysis of repeat elements of the Asian of species-specific reproduction and breeding programmes. For example, there is a remarkable variety of sex-determination systems within aquatic farmed species, and the study of Martínez et al. (2014) highlights various methods of controlling sex ratio with aquaculture breeding programmes. This species diversity also presents an issue for choosing suitable model organisms to inform on the biology of the farmed species of interest. Model finfish species, such as zebrafish, have been well-characterized and Ulloa et al. (2014) highlight their utility for the evaluation of the response to alternative diets. However, due to the vast evolutionary distance between certain farmed aquatic and model species, it is clear that direct research on the species of interest can often be the most feasible and informative.

The aquaculture industry has often been innovative and visionary in their application of new technologies to improve production. Genomics present another major opportunity, and the research published in this special issue provides several excellent examples of their potential or realized application. Using genomic tools to more effectively utilize genetic variation in economically-important traits via sustainable breeding programmes is paramount to the continued successful growth and stability of aquaculture production.

\section{Acknowledgments}

The authors would like to acknowledge funding from Genus plc, CORFO (11IEI-12843 and 12PIE-17669), Government of Chile, Programa U-Inicia, Vicerrectoría de Investigación y Desarrollo, Universidad de Chile, the UK Biotechnology and Biological Sciences Research Council (BBSRC) (BB/H022007/1) and from the Roslin Institute's BBSRC Institute Strategic Funding Grant. seabass (Lates calcarifer) transcriptome and genome. Front. Genet. 5:223. doi: $10.3389 /$ fgene.2014.00223

López, M. E., Neira, R., and Yáñez, J. M. (2015). Applications in the search for genomic selection signatures in fish. Front. Genet. 5:458. doi: 10.3389/fgene.2014.00458

Marancik, D., Gao, G., Paneru, B., Ma, H., Hernandez, A. G., Salem, M., et al. (2014). Whole-body transcriptome of selectively bred, resistant-, control-, and susceptible-line rainbow trout following experimental challenge with Flavobacterium psychrophilum. Front. Genet. 5:453. doi: 10.3389/fgene.2014. 00453

Martínez, P., Viñas, A. M., Sánchez, L., Díaz, N., Ribas, L., and Piferrer, F. (2014). Genetic architecture of sex determination in fish: applications to sex ratio control in aquaculture. Front. Genet. 5:340. doi: 10.3389/fgene.2014. 00340

Ødegård, J., Moen, T., Santi, N., Korsvoll, S. A., Kjøglum, S., and Meuwissen, T. H. E. (2014). Genomic prediction in an admixed population of Atlantic salmon (Salmo salar). Front. Genet. 5:402. doi: 10.3389/fgene.2014.00402

Santos, C. A., Blanck, D. V., and de Freitas, P. D. (2014). RNA-seq as a powerful tool for penaeid shrimp genetic progress. Front. Genet. 5:298. doi: $10.3389 /$ fgene. 2014.00298

Ulloa, P. E., Medrano, J. F., and Feijoo, C. G. (2014). Zebrafish as animal model for aquaculture nutrition research. Front. Genet. 5:313. doi: $10.3389 /$ fgene. 2014.00313

Vandeputte, M., and Haffray, P. (2014). Parentage assignment with genomic markers: a major advance for understanding and exploiting genetic variation of quantitative traits in farmed aquatic animals. Front. Genet. 5:432. doi: 10.3389/fgene.2014.00432 
Yáñez, J. M., Houston, R. D., and Newman, S. (2014). Genetics and genomics of disease resistance in salmonid species. Front. Genet. 5:415. doi: 10.3389/fgene. 2014.00415

Conflict of Interest Statement: The authors declare that the research was conducted in the absence of any commercial or financial relationships that could be construed as a potential conflict of interest.
Copyright (c) 2015 Yáñez, Newman and Houston. This is an open-access article distributed under the terms of the Creative Commons Attribution License (CC $B Y)$. The use, distribution or reproduction in other forums is permitted, provided the original author(s) or licensor are credited and that the original publication in this journal is cited, in accordance with accepted academic practice. No use, distribution or reproduction is permitted which does not comply with these terms. 